\title{
Frequently hypercyclic translation semigroups
}

\author{
Elisabetta M. Mangino \\ Dipartimento di Matematica e Fisica "Ennio De Giorgi" \\ Università del Salento \\ I-73100 Lecce, Italy \\ E-mail: elisabetta.mangino@unisalento.it \\ Marina Murillo Arcila \\ IUMPA, Universitat Politècnica de València \\ Edifici 8G, Camí Vera S/N \\ E-46022 València, Spain \\ E-mail: mamuar1@posgrado.upv.es
}

Dedicated to José Bonet on the occasion of his 60th birthday

\begin{abstract}
Frequent hypercyclicity for translation $C_{0}$-semigroups on weighted spaces of continuous functions is studied. The results are achieved by establishing an analogy between frequent hypercyclicity for translation semigroups and for weighted pseudo-shifts and by characterizing frequently hypercyclic weighted pseudo-shifts on spaces of vanishing sequences. Frequently hypercyclic translation semigroups on

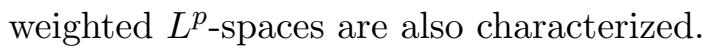

\section{Introduction and preliminaries}

A continuous linear operator $T$ on a separable Banach space $X$ is called $h y$ percyclic if there is an element $x \in X$, called a hypercyclic vector, such that the orbit $\left\{T^{n} x: n \in \mathbb{N}\right\}$ is dense in $X$. The first historically known examples of hypercyclic operators are due to Birkhoff, MacLane and Rolewicz. In

2010 Mathematics Subject Classification: Primary 47A16; Secondary 47D06.

Key words and phrases: frequent hypercyclicity, weighted pseudo-shifts, translation semigroups. 
particular, the last author studied hypercyclicity of weighted shift operators on $l^{p}$ and $c_{0}$. The interest in the study of linear dynamics of shift operators is nowadays still alive, since many classical operators (e.g. derivative operators in spaces of entire functions) can be viewed as such operators. We refer to the recent monographs [11] and [24] for a complete overview on the subject.

In 2005, motivated by Birkhoff's ergodic theorem, Bayart and Grivaux [9] introduced the notion of frequently hypercyclic operators, trying to quantify how "often" an orbit meets non-empty open sets. More precisely, if the lower density of a set $A \subseteq \mathbb{N}$ is defined as

$$
\underline{\operatorname{dens}}(A):=\liminf _{N \rightarrow \infty} \#\{n \leq N: n \in A\} / N,
$$

an operator $T \in L(X)$ is said to be frequently hypercyclic if there exists $x \in X$ (called a frequently hypercyclic vector) such that, for every nonempty open subset $U \subseteq X$,

$$
\underline{\operatorname{dens}}\left(\left\{n \in \mathbb{N}: T^{n} x \in U\right\}\right)>0 .
$$

This notion has been deeply investigated by various authors: see e.g. [22, $14,18]$. In particular frequently hypercyclic weighted shifts have been investigated in $[10,14]$; their behaviour in $l^{p}$ and $c_{0}$ has been completely characterized by Bayart and Ruzsa [12].

In parallel with the theory for linear operators, since the seminal paper by Desch, Schappacher and Webb [20], many researchers turned their attention to the hypercyclic behaviour of strongly continuous semigroups. Actually hypercyclicity appears in solution semigroups of evolution problems associated with "birth and death" equations for cell populations, transport equations, first order partial differential equations, Black-Scholes equation, and diffusion operators like Ornstein-Uhlenbeck operators $[2,4,5,6,8,13$, $15,17,21,25,27]$.

We recall that, if $X$ is a separable infinite-dimensional Banach space, a $C_{0}$-semigroup $\left(T_{t}\right)_{t \geq 0}$ of continuous linear operators on $X$ is said to be hypercyclic if there exists $x \in X$ (called a hypercyclic vector for the semigroup) such that the set $\left\{T_{t} x: t \geq 0\right\}$ is dense in $X$. An element $x \in X$ is said to be a periodic point for the semigroup if there exists $t>0$ such that $T_{t} x=x$. A semigroup $\left(T_{t}\right)_{t \geq 0}$ is called chaotic if it is hypercyclic and the set of periodic points is dense in $X$.

The role of a "test" class, which is played by weighted shifts in the setting of discrete linear dynamical systems, is taken over by translation semigroups in the setting of continuous linear dynamical systems. 
Let $I=\mathbb{R}$ or $I=[0, \infty[$. An admissible weight function on $I$ is a measurable function $\rho: I \rightarrow] 0, \infty[$ for which there exist constants $M \geq 1, \omega \in \mathbb{R}$ such that $\rho(\tau) \leq M e^{\omega t} \rho(\tau+t)$ for all $\tau \in I$ and $t>0$.

If $\rho$ is an admissible weight function, then for every $l>0$ there exist $A, B>0$ such that for all $\sigma \in I$ and $t \in[\sigma, \sigma+l]$,

$$
A \rho(\sigma) \leq \rho(t) \leq B \rho(\sigma+l)
$$

For any $1 \leq p<\infty$, consider the following function spaces:

$$
L_{p}^{\rho}(I)=\left\{u: I \rightarrow \mathbb{R} \mid u \text { is measurable and }\|u\|_{p}^{\rho}<\infty\right\},
$$

where $\|u\|_{p}^{\rho}=\left(\int_{I}|u(t)|^{p} \rho(t) d t\right)^{1 / p}$, and

$$
C_{0}^{\rho}(I)=\left\{u: I \rightarrow \mathbb{R} \mid u \text { is continuous and } \lim _{x \rightarrow \pm \infty} u(x) \rho(x)=0\right\},
$$

with $\|u\|_{\infty}^{\rho}=\sup _{t \in I}|u(t)| \rho(t)$.

If $X$ is any of the spaces above and $\rho$ is an admissible weight function, the translation semigroup $\mathcal{T}=\left(T_{t}\right)_{t \geq 0}$ is defined as usual by

$$
T_{t} f(x)=f(x+t), \quad t \geq 0, f \in X, x \in \mathbb{R},
$$

and it is a $C_{0}$-semigroup (see e.g. [20]).

Hypercyclicity and chaos for translation semigroups have been characterized in $[20,27]$. In particular, if $X$ is one of the spaces $L_{p}^{\rho}(\mathbb{R})$ or $C_{0}^{\rho}(\mathbb{R})$ with an admissible weight function $\rho$, then the translation semigroup $\mathcal{T}$ on $X$ is hypercyclic if and only if for each $\theta \in \mathbb{R}$ there exists a sequence $\left(t_{j}\right)_{j}$ of positive real numbers tending to $\infty$ such that

$$
\lim _{j \rightarrow \infty} \rho\left(t_{j}+\theta\right)=\lim _{j \rightarrow \infty} \rho\left(-t_{j}+\theta\right)=0 .
$$

If $X=C_{0}^{\rho}(\mathbb{R})$, then the translation semigroup $\mathcal{T}$ on $X$ is chaotic if and only if $\lim _{x \rightarrow \pm \infty} \rho(x)=0$.

If $X=L_{p}^{\rho}(\mathbb{R})$, then $\mathcal{T}$ is chaotic if and only if for all $\varepsilon, l>0$ there exists $P>0$ such that

$$
\sum_{k \in \mathbb{Z} \backslash\{0\}} \rho(l+k P)<\varepsilon .
$$

The concept of frequent hypercyclicity was extended to $C_{0}$-semigroups in [3].

The lower density of a measurable set $M \subseteq \mathbb{R}_{+}$is defined by

$$
\underline{\operatorname{Dens}}(M):=\liminf _{N \rightarrow \infty} \mu(M \cap[0, N]) / N
$$


where $\mu$ is the Lebesgue measure on $\mathbb{R}_{+}$.

A $C_{0}$-semigroup $\left(T_{t}\right)_{t \geq 0}$ on a separable Banach space $X$ is said to be frequently hypercyclic if there exists $x \in X$ (called a frequently hypercyclic vector for the semigroup) such that $\underline{\operatorname{Dens}}\left(\left\{t \in \mathbb{R}_{+}: T_{t} x \in U\right\}\right)>0$ for any non-empty open set $U \subseteq X$. In $[16,26]$, it was proved that $x \in X$ is a (frequently) hypercyclic vector for $\left(T_{t}\right)_{t \geq 0}$ if and only if $x$ is a (frequently) hypercyclic vector for each single operator $T_{t}, t>0$. However, this is not the case in general if we consider the chaos property [8].

In [26], a continuous version of the Frequent Hypercyclicity Criterion was proved, based on the Pettis integral and the fact that chaotic translation semigroups on weighted spaces of integrable functions are frequently hypercyclic.

Moreover, in [28], it is proved that the Frequent Hypercyclicity Criterion for semigroups implies the existence of strongly-mixing Borel probability measures with full support.

In this paper we characterize, in the spirit of [12], frequently hypercyclic translation semigroups on $L_{\rho}^{p}(I)$ and, for $\sup _{k} \rho(k+1) / \rho(k)<\infty$, on $C_{0}^{\rho}(I)$. The main results are Theorems 3.2 and 3.9 , proved in the last section. In particular, Theorem 3.2 will be a consequence of Theorem 2.1 which characterizes frequent hypercyclicity of the so-called pseudo-shifts on $c_{0}(I)$ spaces, where $I$ is a countably infinite set.

\section{Frequently hypercyclic weighted pseudo- shift}

We recall the concept of weighted pseudo-shift introduced by Grosse-Erdmann [23].

Given topological sequence spaces $X, Y$ over countably infinite sets $I$ and $J$ respectively, a continuous linear operator $T: X \rightarrow Y$ is called a weighted pseudo-shift if there is a sequence $\left(b_{j}\right)_{j \in J}$ of non-zero scalars and an injective mapping $\phi: J \rightarrow I$ such that

$$
T\left[\left(x_{i}\right)_{i \in I}\right]=\left(b_{j} x_{\phi(j)}\right)_{j \in J} \quad \text { for }\left(x_{i}\right)_{i \in I} \in X .
$$

We will be interested in weighted pseudo-shifts acting on spaces of vanishing sequences. More precisely, given a countable set $I$, we consider the space

$$
c_{0}(I)=\left\{\left(x_{i}\right)_{i \in I} \in \mathbb{R}^{I} \mid \forall \varepsilon>0 \exists J \subseteq I, J \text { finite } \forall i \in I \backslash J:\left|x_{i}\right|<\varepsilon\right\},
$$


endowed with the norm $\left\|\left(x_{i}\right)_{i \in I}\right\|=\sup _{i \in I}\left|x_{i}\right|$.

Obviously, if $\left(W_{p}\right)_{p \in \mathbb{N}}$ is an increasing sequence of finite subsets of $I$ such that $I=\bigcup_{p=1}^{\infty} W_{p}$, then

$$
c_{0}(I)=\left\{\left(x_{i}\right)_{i \in I} \in \mathbb{R}^{I}\left|\forall \varepsilon>0 \exists n \in \mathbb{N} \forall i \in I \backslash W_{n}:\right| x_{i} \mid<\varepsilon\right\} .
$$

The first result that we prove is a characterization of frequently universal sequences of weighted pseudo-shifts on $c_{0}(I)$.

We recall that a sequence $\left(T_{n}\right)_{n \in \mathbb{N}}$ of continuous mappings between topological spaces $X$ and $Y$ is said to be frequently universal if there exists $x \in X$, called a frequently universal vector for the sequence, such that for every non-empty open set $U \subseteq Y$,

$$
\underline{\operatorname{dens}}\left(\left\{n \in \mathbb{N}: T_{n} x \in U\right\}\right)>0 .
$$

Following the idea of Bayart and Ruzsa [12] for weighted backward shifts on $c_{0}(\mathbb{Z})$, we first obtain a characterization for weighted pseudo-shifts.

Theorem 2.1. Let $\left(T_{n}\right)_{n \in \mathbb{N}}$ be a sequence of weighted pseudo-shifts on $c_{0}(I)$ defined by $T_{n}\left[\left(x_{i}\right)_{i \in I}\right]=\left(b_{i}^{n} x_{\phi_{n}(i)}\right)_{i \in I}$, where the $b_{i}^{n}$ are positive real numbers. Assume that:

(i) $\left(\phi_{n}\right)_{n}$ is a run-away sequence, i.e. for any finite subsets $I_{0}, J_{0} \subseteq I$ there exists $n_{0} \in \mathbb{N}$ such that, for every $n \geq n_{0}, \phi_{n}\left(J_{0}\right) \cap I_{0}=\emptyset$,

(ii) there exists $\rho>1$ such that $1 / \rho^{|n-m|} \leq b_{s}^{n} / b_{t}^{m}$ for all $n, m \in \mathbb{N}$ and $s, t \in I$ such that $\phi_{n}(s)=\phi_{m}(t)$,

(iii) there exists $g: I \rightarrow \mathbb{R}$ such that $|n-m| \leq|g(s)-g(t)|$ for all $n, m \in \mathbb{N}$ and $s, t \in I$ such that $\phi_{n}(s)=\phi_{m}(t)$,

(iv) $\left(W_{p}\right)_{p \in \mathbb{N}}$ is an increasing sequence of finite subsets of $I$ such that $I=\bigcup_{p=1}^{\infty} W_{p}$.

Then $\left(T_{n}\right)_{n \in \mathbb{N}}$ is frequently universal on $c_{0}(I)$ if and only if there exist a sequence $(M(p))_{p \in \mathbb{N}}$ of positive real numbers tending to $\infty$ and a sequence $\left(E_{p}\right)_{p \in \mathbb{N}}$ of subsets of $\mathbb{N}$ such that:

(a) for any $p \geq 1$, $\underline{\operatorname{dens}}\left(E_{p}\right)>0$,

(b) for any distinct $p, q \geq 1, n \in E_{p}$ and $m \in E_{q}, \phi_{n}\left(W_{p}\right) \cap \phi_{m}\left(W_{q}\right)=\emptyset$,

(c) for every $p \geq 1$ and every $s \in W_{p}, \lim _{n \rightarrow \infty, n \in E_{p}} b_{s}^{n}=\infty$, 
(d) for any $p, q \geq 1, n \in E_{p}, m \in E_{q}$ with $n \neq m, t \in W_{q}$ and $s \in I$ such that $\phi_{n}(s)=\phi_{m}(t)$,

$$
\frac{b_{s}^{n}}{b_{t}^{m}} \leq \frac{1}{M(p) M(q)}
$$

Moreover, one can replace "there exists a sequence $(M(p))_{p \in \mathbb{N}}$ and a sequence $\left(E_{p}\right)_{p \in \mathbb{N}}$ " by "for any sequence $(M(p))_{p \in \mathbb{N}}$ there exists a sequence $\left(E_{p}\right)_{p \in \mathbb{N}}$ ".

Proof. We first observe that if properties (a) to (d) hold for some sequence $(M(p))_{p \in \mathbb{N}}$, then they are also satisfied for any sequence $(M(p))_{p \in \mathbb{N}}$, on considering, if necessary, a subsequence of $\left(E_{p}\right)_{p \in \mathbb{N}}$.

" $\Rightarrow$ ": Let $x \in c_{0}(I)$ be a frequently universal vector for $\left(T_{n}\right)_{n \in \mathbb{N}}$. Let $\left(\alpha_{p}\right)_{p \in \mathbb{N}}$ be a strictly increasing sequence of positive real numbers such that $\alpha_{1}=2$ and for all $p \geq 2, \alpha_{p}>4 \alpha_{p-1} \rho^{2 \Psi(p)}$, where $\Psi(p)=\max \{|g(t)|$ : $\left.t \in W_{p}\right\}$. Define

$$
F_{p}=\left\{n \in \mathbb{N}:\left\|T_{n} x-\alpha_{p} \sum_{i \in W_{p}} e_{i}\right\|<1 / p\right\} .
$$

If $F_{p}=\left\{n_{k}^{p}: k \in \mathbb{N}\right\}$, where $\left(n_{k}^{p}\right)_{k \in \mathbb{N}}$ is an increasing sequence of natural numbers, we define $E_{p}=\left\{n_{(2[\Psi(p)]+3) k}^{p}: k \in \mathbb{N}\right\}$ where $[\Psi(p)]$ is the integer part of $\Psi(p)$.

Clearly dens $\left(E_{p}\right)>0$ and the distance between two different elements of $E_{p}$ is greater than $2 \Psi(p)$. Moreover

$$
\forall p \in \mathbb{N} \forall s \in W_{p} \forall n \in E_{p}: \quad \alpha_{p} / 2 \leq\left|b_{s}^{n} x_{\phi_{n}(s)}\right|<2 \alpha_{p} .
$$

Indeed, $b_{s}^{n} x_{\phi_{n}(s)}$ is the $s$ th coefficient of $T_{n} x$, so

$$
\left|b_{s}^{n} x_{\phi_{n}(s)}\right| \leq\left\|T_{n} x-\alpha_{p} \sum_{i \in W_{p}} e_{i}\right\|+\alpha_{p}\left\|\sum_{i \in W_{p}} e_{i}\right\|<\frac{1}{p}+\alpha_{p}<2 \alpha_{p},
$$

while

$$
\begin{aligned}
\left|b_{s}^{s} x_{\phi_{n}(s)}\right| & \geq \alpha_{p}-\left|b_{s}^{n} x_{\phi_{n}(s)}-\alpha_{p}\right| \geq \alpha_{p}-\left\|T_{n} x-\alpha_{p} \sum_{i \in W_{p}} e_{i}\right\| \\
& \geq \alpha_{p}-1 / p \geq \alpha_{p} / 2 .
\end{aligned}
$$

In particular,

$$
\forall p \in \mathbb{N} \forall s \in W_{p} \forall n \in E_{p}: \quad x_{\phi_{n}(s)} \neq 0 .
$$

In order to prove (b), fix $p \neq q$, with $p<q, n \in E_{p}, m \in E_{q}$ and assume by contradiction, that there exist $s \in W_{p}$ and $t \in W_{q}$ such that $\phi_{n}(s)=\phi_{m}(t)$. Then, by $(2.2)$,

$$
\frac{1}{\rho^{2 \Psi(q)}} \leq \frac{1}{\rho^{|n-m|}} \leq \frac{\left|b_{s}^{n} x_{\phi_{n}(s)}\right|}{\left|b_{t}^{m} x_{\phi_{m}(t)}\right|} \leq 2 \alpha_{p} \frac{2}{\alpha_{q}} \leq 4 \frac{\alpha_{q-1}}{\alpha_{q}}
$$


contradicting the choice of $\left(\alpha_{p}\right)_{p}$.

Now let $p \geq 1$ and $s \in W_{p}$. Let $M>0$. Given $\varepsilon=\alpha_{p} /(2 M)$, since $x \in c_{0}(I)$, there exists $J \subseteq I$ finite such that $\left|x_{i}\right|<\varepsilon$ for all $i \in I \backslash J$. Since $\left(\phi_{n}\right)$ is a run-away sequence, there exists $n_{0} \in \mathbb{N}$ such that for all $n \in \mathbb{N}$ with $n>n_{0}$ and all $s \in W_{p}$ we have $\phi_{n}(s) \notin J$, and so $\left|x_{\phi_{n}(s)}\right|<\varepsilon$. Hence, for all $n \in E_{p}$ with $n \geq n_{0}$, by (2.2) and (2.4),

$$
b_{s}^{n} \geq \frac{\alpha_{p}}{2\left|x_{\phi_{n}(s)}\right|} \geq \frac{\alpha_{p}}{2 \varepsilon}=M .
$$

So, we have proved (c).

Finally, let $p, q \geq 1, n \in E_{p}, m \in E_{q}$ with $n \neq m, t \in W_{q}$ and $s \in I$ be such that $\phi_{n}(s)=\phi_{m}(t)$. Then $p \neq q$, as otherwise, since $n \neq m$, by the definition of $E_{p}$ we have $|n-m|>2 \Psi(p)$; on the other hand, (iii) yields $|n-m| \leq 2 \Psi(p)$, a contradiction. Therefore, since $p \neq q$, we can apply (b) to get $s \notin W_{p}$ and so the $s$-coefficient of $T_{n} x-\alpha_{p} \sum_{i \in W_{p}} e_{i}$ is $b_{s}^{n} x_{\phi_{n}(s)}$. Hence, by (2.2),

$$
\frac{b_{s}^{n}}{b_{t}^{m}}=\frac{\left|b_{s}^{n} x_{\phi_{n}(s)}\right|}{\left|b_{t}^{m} x_{\phi_{m}(t)}\right|} \leq \frac{1}{p} \frac{2}{\alpha_{q}} \leq \frac{1}{p} \frac{1}{q} .
$$

Hence (d) holds with $M(p)=p$.

" $\Leftarrow$ ": We first observe that if (b) holds, then

$$
\forall p, q \in \mathbb{N}, p \neq q: \quad E_{p} \cap E_{q}=\emptyset .
$$

Indeed, assume $p<q$; if there exists $n \in E_{p} \cap E_{q}$, then for any $s \in W_{p} \subseteq W_{q}$, one gets $\phi_{n}(s) \in \phi_{n}\left(W_{p}\right) \cap \phi_{n}\left(W_{q}\right)$, contradicting (b).

As properties (a) to $(\mathrm{d})$ hold true for any sequence $(M(p))_{p \in \mathbb{N}}$, we may assume that $M(p) \geq \rho^{4 p}$ for any $p \geq 1$.

We set

$$
E_{p}^{\prime}=E_{p} \backslash \bigcup_{s \in W_{p}}\left\{n \in \mathbb{N}: b_{s}^{n} \leq \rho^{4 p}\right\} .
$$

By (c), $E_{p}^{\prime}$ is a cofinite subset of $E_{p}$, hence $\underline{\operatorname{dens}}\left(E_{p}^{\prime}\right)>0$. If $E_{p}^{\prime}=\left\{n_{k}^{p}: k \in \mathbb{N}\right\}$, where $\left(n_{k}^{p}\right)_{k}$ is an increasing sequence of natural numbers, we consider the set $G_{p}=\left\{n_{(2[\Psi(p)]+3) k}^{p}: k \in \mathbb{N}\right\}$. It has positive lower density and moreover the distance between two different elements of $G_{p}$ is greater than $2 \Psi(p)$.

Let $\left(y^{p}\right)_{p \geq 0}$ be a dense sequence in $c_{0}(I)$ such that $\operatorname{supp}\left(y^{p}\right) \subseteq W_{p}$ and $\left\|y^{p}\right\|<\rho^{p}$. We define $x \in \mathbb{R}^{I}$ by setting

$$
x_{i}= \begin{cases}\frac{1}{b_{s}^{n}} y^{p}(s) & \text { if } i=\phi_{n}(s), n \in G_{p}, s \in W_{p}, \\ 0 & \text { otherwise. }\end{cases}
$$


This definition is correct, because if $i=\phi_{n}(s)=\phi_{m}(t)$ with $n \in G_{p}$, $s \in W_{p}, m \in G_{q}$ and $t \in W_{q}$, then, by (b), $p=q$, and assumption (iii) yields $|n-m| \leq|g(s)-g(t)| \leq 2 \Psi(p)$; hence, by the definition of $G_{p}, n=m$ and so $s=t$, by the injectivity of $\phi_{n}$.

We have $x \in c_{0}(I)$. Indeed, given $\varepsilon>0$, there exists $p_{0} \in \mathbb{N}$ such that for $p \geq p_{0}$ and $n \in G_{p}, s \in W_{p}, i=\phi_{n}(s)$,

$$
\left|x_{i}\right| \leq \frac{\rho^{p}}{\rho^{4 p}} \leq \varepsilon
$$

If $p \leq p_{0}$, then

$$
\left|x_{i}\right| \leq \frac{\rho^{p_{0}}}{b_{s}^{n}} \rightarrow 0 \quad \text { as } n \rightarrow \infty .
$$

We finally show that $x$ is a frequently hypercyclic vector by proving that for all $p \geq 1$ and $n \in G_{p},\left\|T_{n} x-y^{p}\right\|<\varepsilon(p)$ with $\varepsilon(p) \rightarrow 0$ as $p \rightarrow \infty$. We have

$$
\left\|T_{n} x-y^{p}\right\|=\sup _{s \notin W_{p}}\left|b_{s}^{n} x_{\phi_{n}(s)}\right| .
$$

If $s \notin W_{p}$, then $b_{s}^{n} x_{\phi_{n}(s)}$ does not vanish if and only if

$$
\exists q \geq 1 \exists m \in G_{q}, t \in W_{q}: \quad \phi_{n}(s)=\phi_{m}(t) .
$$

If (2.7) holds, then $n \neq m$, as otherwise, $p=q$ by (2.5) and $s=t$ by the injectivity of $\phi_{n}$, which is impossible since $s \notin W_{p}$ and $t \in W_{p}=W_{q}$.

Hence, we can apply (d) to get

$$
\left|b_{s}^{n} x_{\phi_{n}(s)}\right|=\left|\frac{b_{s}^{n}}{b_{t}^{m}} y^{q}(t)\right| \leq \frac{\rho^{q}}{M(p) M(q)} \leq \frac{\rho^{q}}{\rho^{p} \rho^{q}}=\frac{1}{\rho^{p}} . \square
$$

As a corollary, we obtain a characterization of frequent hypercyclicity for weighted backward shift operators defined on $c_{0}(I)$ where $I \subseteq \mathbb{R}$.

Corollary 2.2. Let I be a countably infinite subset of $\mathbb{R}$ such that $I+\mathbb{Z} \subseteq I$ (resp. $I+\mathbb{N} \subseteq I), I=\bigcup_{p=1}^{\infty} W_{p}$, where $\left(W_{p}\right)_{p}$ is an increasing sequence of finite subsets. Let $\left(w_{i}\right)_{i \in I}$ be a family of positive real numbers such that

$$
0<\inf _{i \in I} w_{i} \leq \sup _{i \in I} w_{i}<\infty .
$$

The operator $T: c_{0}(I) \rightarrow c_{0}(I)$ defined by $T\left(x_{i}\right)_{i \in I}=\left(w_{i} x_{i+1}\right)_{i \in I}$ is frequently hypercyclic on $c_{0}(I)$ if and only if there exist a sequence $(M(p))_{p \in \mathbb{N}}$ of positive real numbers tending to $\infty$ and a sequence $\left(E_{p}\right)_{p \in \mathbb{N}}$ of subsets of $\mathbb{N}$ such that

(a) for any $p \geq 1$, $\underline{\operatorname{dens}}\left(E_{p}\right)>0$, 
(b) for any $p, q \geq 1, p \neq q,\left(E_{p}+W_{p}\right) \cap\left(E_{q}+W_{q}\right)=\emptyset$,

(c) for all $p \geq 1$ and $s \in W_{p}, \lim _{n \rightarrow \infty, n \in E_{p}} w_{s} \ldots w_{s+n-1}=\infty$,

(d) for any $p, q \geq 1, n \in E_{p}$ and $m \in E_{q}$ with $m \neq n$, and $t \in W_{q}$ (resp. $t \in W_{q}$ such that $\left.t+(m-n) \in I\right)$,

$$
\frac{w_{m-n+t} \ldots w_{m+t-1}}{w_{t} \ldots w_{t+m-1}} \leq \frac{1}{M(p) M(q)}
$$

Moreover, one can replace "there exist a sequence $(M(p))_{p \in \mathbb{N}}$ and a sequence $\left(E_{p}\right)_{p \in \mathbb{N}}$ " by "for any sequence $(M(p))_{p \in \mathbb{N}}$ there exists a sequence $\left(E_{p}\right)_{p \in \mathbb{N}}$ ".

Proof. Observe that $T$ is frequently hypercyclic if and only the family of its powers $\left(T^{n}\right)_{n \in \mathbb{N}}$ is frequently universal. If we set $T_{n}=T^{n}$, we have that

$$
T_{n}\left[\left(x_{i}\right)_{i \in I}\right]=\left(w_{i} w_{i+1} \ldots w_{i+n-1} x_{i+n}\right)_{i \in I} .
$$

Therefore, if we set

$$
b_{s}^{n}:=w_{s} w_{s+1} \ldots w_{s+n-1}, \quad \phi(s):=s+1, \quad \phi_{n}:=\underbrace{\phi \circ \cdots \circ \phi}_{n}
$$

and $g(s):=s$ for all $s \in I$ and $n \in \mathbb{N}$, then assumptions (i), (iii) and (iv) of Theorem 2.1 are trivially satisfied, while (ii) follows from (2.8). The characterization follows by Theorem 2.1.

Remark 2.3. Observe that condition (d) is equivalent to saying that for any $p, q \geq 1, n \in E_{p}$ and $m \in E_{q}$ with $n \neq m$, and $t \in W_{q}$ (resp. $t \in W_{q}$ such that $t+(m-n) \in I)$,

$$
\begin{cases}w_{t} \ldots w_{t+m-n-1} \geq M(p) M(q) & \text { if } m>n \\ w_{t+(m-n)} \ldots w_{t-2} w_{t-1} \leq \frac{1}{M(p) M(q)} & \text { if } m<n\end{cases}
$$

and we obtain the conditions of [12, Theorem 12].

\section{$3 \quad$ Frequently hypercyclic translation semigroups}

The purpose of this section is to obtain a characterization of frequent hypercyclicity for translation semigroups on $C_{0}^{\rho}(\mathbb{R})$ under the assumption $\sup _{k \in \mathbb{Z}} \rho(k+1) / \rho(k)<\infty$, and on $L_{p}^{\rho}(\mathbb{R})$.

In the following we set, for any $r, s \in \mathbb{Z}, \llbracket r, s \rrbracket=[r, s] \cap \mathbb{Z}$.

To treat the case of continuous functions, we recall some known results about the construction of a Schauder basis in $C_{0}(\mathbb{R})$, referring for more details to [29]. 
Let $\widetilde{D}$ be the set of dyadic numbers except 1 , that is, $\widetilde{D}=\bigcup_{n=0}^{\infty} D_{n}$ where $D_{0}=\{0\}$ and, if $n \geq 1$,

$$
D_{n}=\left\{\frac{2 k-1}{2^{n}}: k=1, \ldots, 2^{n-1}\right\}
$$

For any $\tau \in D_{n}$, set $\tau^{-}=\tau-2^{-n}$ and $\tau^{+}=\tau+2^{-n}$.

Let $\varphi(x):=\max \{0,1-|x|\}$ for $x \in \mathbb{R}$, and define for every $k \in \mathbb{Z}$, $\tau \in D_{n}$, and $x \in \mathbb{R}$,

$$
\varphi_{k+\tau}(x):=\varphi\left(2^{n}(x-k-\tau)\right) .
$$

Observe that $\varphi_{k+\tau}(x)=\varphi_{\tau}(x-k)$ where $\varphi_{\tau}$ is the Faber-Schauder dyadic function with peak at $\tau$.

Set $I=\mathbb{Z}+\widetilde{D}$ and consider the partition $I=\bigcup_{n \geq 0} V_{n}$ where $V_{0}=\{0\}$, and

$$
V_{n}=\left\{-n+h+D_{h}: h=0,1, \ldots, n\right\} \cup\left\{h+D_{n-h}: h=1, \ldots, n\right\} .
$$

We define an order on $I$ assuming that the elements of $V_{k}$ are earlier than those in $V_{n}$ if $0 \leq k<n$, and within each $V_{n}$ we keep the usual order.

The system $\left(\varphi_{i}\right)_{i \in I}$ is a Schauder basis in $C_{0}(\mathbb{R})$. More precisely, if $f \in C_{0}(\mathbb{R})$, then $f=\sum_{k+\tau \in \mathbb{Z}+\widetilde{D}} a_{k+\tau} \varphi_{k+\tau}$ where

$$
a_{k+\tau}= \begin{cases}f(k), & k \in \mathbb{Z}, \tau=0, \\ f(k+\tau)-\frac{1}{2}\left(f\left(k+\tau^{-}\right)+f\left(k+\tau^{+}\right)\right), & k \in \mathbb{Z}, \tau \neq 0 .\end{cases}
$$

By the construction of the functions $\varphi_{k+\tau}$, it follows that for any $n \in \mathbb{Z}_{+}$, for every family $\left(a_{k+\tau}\right)_{k+\tau \in \mathbb{Z}+D_{n}}$ of real numbers, and for every $x \in \mathbb{R}$ :

$$
\sum_{k \in \mathbb{Z}, \tau \in D_{n}}\left|a_{k+\tau} \varphi_{k+\tau}(x)\right| \leq 2 \sup _{k \in \mathbb{Z}, \tau \in D_{n}}\left|a_{k+\tau}\right| .
$$

If we set for every $n \in \mathbb{Z}_{+}$

$$
\widetilde{D}_{n}:=\bigcup_{h=0}^{n} D_{h}, \quad W_{n}=\llbracket-n, n \rrbracket+\widetilde{D}_{n},
$$

then clearly $\left(W_{n}\right)_{n \geq 0}$ is an increasing sequence of finite subsets such that $\mathbb{Z}+\widetilde{D}=\bigcup_{n \geq 0} W_{n}$. Thus

$$
\begin{aligned}
& c_{0}(\mathbb{Z}+\widetilde{D})=\left\{\left(a_{k+\tau}\right)_{k, \tau} \in \mathbb{R}^{\mathbb{Z}+\widetilde{D}}\left|\forall \varepsilon>0 \exists n \in \mathbb{N}: k+\tau \notin W_{n} \Rightarrow\right| a_{k+\tau} \mid<\varepsilon\right\} \\
& =\left\{\left(a_{k+\tau}\right)_{k, \tau} \in \mathbb{R}^{\mathbb{Z}+\widetilde{D}} \mid \forall \varepsilon>0 \exists n \in \mathbb{N}:\left(|k|>n \text { or } \tau \notin \widetilde{D}_{n}\right) \Rightarrow\left|a_{k+\tau}\right|<\varepsilon\right\} .
\end{aligned}
$$

For any $x \in \mathbb{R}$, let $[x]$ denote the integer part of $x$. 
Lemma 3.1. Let $\rho$ be an admissible weight function on $\mathbb{R}$ such that $\rho(x)=$ $\rho([x])$ for any $x \in \mathbb{R}$ and let $T_{1}: C_{0}^{\rho}(\mathbb{R}) \rightarrow C_{0}^{\rho}(\mathbb{R})$ be the translation operator defined as $T_{1} f(x)=f(x+1)$. Then $T_{1}$ is quasiconjugate to the weighted backward shift operator $B_{w}: c_{0}(\mathbb{Z}+\widetilde{D}) \rightarrow c_{0}(\mathbb{Z}+\widetilde{D})$ defined by

$$
B_{w}\left[\left(x_{k+\tau}\right)_{k+\tau \in \mathbb{Z}+\widetilde{D}}\right]=\left(w_{k+\tau} x_{k+\tau+1}\right)_{k+\tau \in \mathbb{Z}+\widetilde{D}}, f
$$

where

$$
w_{k+\tau}:=\frac{\rho(k+\tau)}{\rho(k+1+\tau)}=\frac{\rho(k)}{\rho(k+1)}, \quad k+\tau \in \mathbb{Z}+\widetilde{D} .
$$

Moreover, $B_{w}$ is quasiconjugate to $T_{1}$.

Proof. To prove that $T_{1}$ is quasiconjugate to $B_{w}$, we exhibit a continuous linear operator $Q: C_{0}^{\rho}(\mathbb{R}) \rightarrow c_{0}(\mathbb{Z}+\widetilde{D})$ with dense range such that the following diagram commutes:

$$
\begin{array}{cccc}
C_{0}^{\rho}(\mathbb{R}) & \stackrel{T_{1}}{\longrightarrow} & C_{0}^{\rho}(\mathbb{R}) \\
\downarrow Q & & \downarrow Q \\
c_{0}(\mathbb{Z}+\widetilde{D}) \stackrel{B_{w}}{\longrightarrow} & c_{0}(\mathbb{Z}+\widetilde{D})
\end{array}
$$

Given $f \in C_{0}^{\rho}(\mathbb{R})$, we define $Q(f)=\left(a_{k+\tau}\right)_{k+\tau \in \mathbb{Z}+\widetilde{D}}$ where

$$
a_{k+\tau}=\rho(k) \cdot \begin{cases}f(k), & k \in \mathbb{Z}, \tau=0 \\ f(k+\tau)-\frac{1}{2}\left(f\left(k+\tau^{-}\right)+f\left(k+\tau^{+}\right)\right), & k \in \mathbb{Z}, \tau \neq 0\end{cases}
$$

It holds that $Q(f) \in c_{0}(\mathbb{Z}+\widetilde{D})$. Indeed, since $f \in C_{0}^{\rho}(\mathbb{R})$, for every $\varepsilon>0$,

(1) there exists $N_{1} \in \mathbb{N}$ such that if $|x|>N_{1}$, then $|f(x) \rho(x)|<\frac{\varepsilon}{2}$

(2) there exists $\delta>0$ such that, for all $x, y \in\left[-N_{1}-1, N_{1}+2\right]$, if $|x-y|<\delta$

then $|f(x)-f(y)|<\frac{\varepsilon}{\rho_{N_{1}}}$, where $\rho_{N_{1}}=\max _{k \in \llbracket-N_{1}-1, N_{1}+2 \rrbracket} \rho(k)$.

Choose $N_{2} \in \mathbb{N}$ such that $\frac{1}{2^{N_{2}}}<\delta$ and set $N=\max \left\{N_{1}+1, N_{2}\right\}$. Let $k+\tau \in(\mathbb{Z}+\widetilde{D}) \backslash W_{N}$.

If $|k|>N_{1}+1$, by (1) it is clear that for every $s \in[0,1[$,

$$
|f(k+s) \rho(k)|=|f(k+s) \rho(k+s)|<\frac{\varepsilon}{2},
$$

since $|k+s|>N_{1}$. Hence, by the continuity of $f$, for every $s \in[0,1]$,

$$
|f(k+s) \rho(k)| \leq \frac{\varepsilon}{2} .
$$

By replacing $s$ by $\tau, \tau^{+}$and $\tau^{-}$in (3.4), we immediately get $\left|a_{k+\tau}\right| \leq \varepsilon$. 
If $|k| \leq N_{1}+1$, then necessarily $\tau \in D_{n}$, with $n>N$, and, in particular, $\tau \neq 0$. It holds that

$$
\left|\tau-\tau^{-}\right|<\frac{1}{2^{N}}<\delta,\left|\tau-\tau^{+}\right|<\frac{1}{2^{N}}<\delta,
$$

and $k+\tau, k+\tau^{-}, k+\tau^{+} \in\left[-N_{1}-1, N_{1}+2\right]$, thus, by (2) we get that

$$
\left|a_{k+\tau}\right|=\left|\rho(k)\left(f(k+\tau)-\frac{1}{2}\left(f\left(k+\tau^{-}\right)+f\left(k+\tau^{+}\right)\right)\right)\right| \leq \varepsilon .
$$

Then $Q: C_{0}^{\rho}(\mathbb{R}) \rightarrow c_{0}(\mathbb{Z}+\widetilde{D})$ is well-defined and clearly linear. Moreover, for every $f \in C_{0}^{\rho}(\mathbb{R}), k \in \mathbb{Z}$, we have

$$
|f(k+s) \rho(k)|=|f(k+s) \rho(k+s)| \leq\|f\|_{\infty}^{\rho} \quad \text { for all } s \in[0,1[,
$$

hence, by the continuity of $f$,

$$
|f(k+s) \rho(k)| \leq\|f\|_{\infty}^{\rho} \quad \text { for all } s \in[0,1] .
$$

By replacing $s$ by $\tau, \tau^{+}$and $\tau^{-}$in (3.5), with $\tau \in \widetilde{D}$, we immediately get $\|Q(f)\| \leq 2\|f\|_{\infty}^{\rho}$, so $Q$ is continuous.

To prove that $Q$ has dense range, it is enough to show that for every $h \in \mathbb{Z}$ and $\sigma \in \widetilde{D}$,

$$
\left(x_{k+\tau}^{h+\sigma}\right)_{k \in \mathbb{Z}, \tau \in \widetilde{D}} \in Q\left(C_{0}^{\rho}(\mathbb{R})\right) \quad \text { where } \quad x_{k+\tau}^{h+\sigma}=\delta_{(h, \sigma)}(k, \tau),
$$

and, indeed, by the definition of $\varphi_{h+\sigma}$, we have

$$
Q\left(\rho(h)^{-1} \varphi_{h+\sigma}\right)=\left(x_{k+\tau}^{h+\sigma}\right)_{k \in \mathbb{Z}, \tau \in \widetilde{D}} .
$$

Finally, by observing that, for every $f \in C_{0}^{\rho}(\mathbb{R}), Q \circ T_{1}(f)=\left(b_{k+\tau}\right)_{k+\tau \in \mathbb{Z}+\widetilde{D}}$, where

$$
b_{k+\tau}=\left\{\begin{array}{lr}
f(k+1) \rho(k)=a_{k+1} \frac{\rho(k)}{\rho(k+1)}, & \text { if } \tau=0, \\
\left(f(k+1+\tau)-\frac{1}{2}\left(f\left(k+1+\tau^{-}\right)+f\left(k+1+\tau^{+}\right)\right) \rho(k)\right. & \text { if } \tau \neq 0,
\end{array}\right.
$$

for $k \in \mathbb{Z}$ and $\tau \in \widetilde{D}$, it is immediate that $Q \circ T_{1}(f)=B_{w} \circ Q(f)$.

Now, let us prove that $B_{w}$ is quasiconjugate to $T_{1}$, namely that there exists a continuous linear operator $P: c_{0}(\mathbb{Z}+\widetilde{D}) \rightarrow C_{0}^{\rho}(\mathbb{R})$ with dense range such that the following diagram commutes:

$$
\begin{array}{ccc}
c_{0}(\mathbb{Z}+\widetilde{D}) & \stackrel{B_{w}}{\longrightarrow} & c_{0}(\mathbb{Z}+\widetilde{D}) \\
\downarrow P & & \downarrow P \\
C_{0}^{\rho}(\mathbb{R}) & \stackrel{T_{1}}{\longrightarrow} & C_{0}^{\rho}(\mathbb{R})
\end{array}
$$


Given $\left(a_{k+\tau}\right)_{k \in \mathbb{Z}, \tau \in \widetilde{D}} \in c_{0}(\mathbb{Z}+\widetilde{D})$, we define

$$
P\left(\left(a_{k+\tau}\right)_{k \in \mathbb{Z}, \tau \in \tilde{D}}\right)=\sum_{n=0}^{\infty} \frac{1}{2^{n}}\left(\sum_{k \in \mathbb{Z}, \tau \in D_{n}} \frac{a_{k+\tau}}{\rho(k)} \varphi_{k+\tau}\right) .
$$

Observe that, for every $N \in \mathbb{N}, x \in[-N, N]$, taking (3.3) into account,

$$
\begin{aligned}
& \left|\sum_{k \in \mathbb{Z}, \tau \in D_{n}} \frac{a_{k+\tau}}{\rho(k)} \varphi_{k+\tau}(x)\right| \leq\left|\sum_{|k| \leq N, \tau \in D_{n}} \frac{a_{k+\tau}}{\rho(k)} \varphi_{k+\tau}(x)\right| \leq \\
& \leq \sum_{|k| \leq N, \tau \in D_{n}} \frac{\left|a_{k+\tau}\right|}{\rho(k)} \varphi_{k+\tau}(x) \leq 2 \frac{\left\|\left(a_{k+\tau}\right)_{k, \tau}\right\|}{\min _{|k| \leq N}\{\rho(k)\}},
\end{aligned}
$$

thus by the Weierstrass M-test, the series on the right-hand side of (3.6) is uniformly convergent on $[-N, N]$. It follows that $P\left(\left(a_{k+\tau}\right)_{k \in \mathbb{Z}, \tau \in \widetilde{D}}\right)$ is a continuous function on $\mathbb{R}$.

Moreover, for every $x \in \mathbb{R}$ there exists $\bar{k} \in \mathbb{Z}$ such that $\bar{k} \leq x<\bar{k}+1$. Hence, by (3.3) and by (1.1) applied with $l=1$

$$
\begin{array}{r}
\left|P\left(\left(a_{k+\tau}\right)_{k, \tau}\right)(x) \rho(x)\right|=\left|\rho(x) \sum_{n=0}^{\infty} \frac{1}{2^{n}}\left(\sum_{k \in \mathbb{Z}, \tau \in D_{n}} \frac{a_{k+\tau}}{\rho(k)} \varphi_{k+\tau}(x)\right)\right|= \\
=\left|\rho(\bar{k})\left(\sum_{k \in \mathbb{Z}} \frac{a_{k}}{\rho(k)} \varphi_{k}(x)\right)+\rho(\bar{k}) \sum_{n=1}^{\infty} \frac{1}{2^{n}}\left(\sum_{k \in \mathbb{Z}, \tau \in D_{n}} \frac{a_{k+\tau}}{\rho(k)} \varphi_{k+\tau}(x)\right)\right| \\
=\left|a_{\bar{k}} \varphi_{\bar{k}}(x)+\frac{\rho(\bar{k})}{\rho(\bar{k}+1)} a_{\bar{k}+1} \varphi_{\bar{k}+1}(x)+\rho(\bar{k}) \sum_{n=1}^{\infty} \frac{1}{2^{n}}\left(\sum_{\tau \in D_{n}} \frac{a_{\bar{k}+\tau}}{\rho(\bar{k})} \varphi_{\bar{k}+\tau}(x)\right)\right| \leq \\
\leq \frac{\rho(\bar{k})}{\rho(\bar{k}+1)}\left|a_{\bar{k}+1}\right|+\left|\sum_{n=0}^{\infty} \frac{1}{2^{n}}\left(\sum_{\tau \in D_{n}} a_{\bar{k}+\tau} \varphi_{\bar{k}+\tau}(x)\right)\right| \leq \\
\leq B\left|a_{\bar{k}+1}\right|+2 \sum_{n=0}^{\infty} \frac{1}{2^{n}} \sup _{\tau \in D_{n}}\left|a_{\bar{k}+\tau}\right| .
\end{array}
$$

Thus, if $\varepsilon>0$ and $N \in \mathbb{N}$ is such that $\left|a_{k+\tau}\right|<\varepsilon$ if $k+\tau \notin W_{N}$, then for any $x \in \mathbb{R}$ such that $|x|>N+1$, it holds that $|\bar{k}|>N$ and $|\bar{k}+1|>N$, thus $\bar{k}+\tau \notin W_{N}$ for every $\tau \in \widetilde{D}$ and $\bar{k}+1 \notin W_{N}$. Therefore $\left|a_{\bar{k}+\tau}\right|<\varepsilon$ and $\left|a_{\bar{k}+1}\right|<\varepsilon$. Hence

$$
\left|P\left(\left(a_{k+\tau}\right)_{k, \tau}\right)(x) \rho(x)\right|<(B+4) \varepsilon .
$$

On the other hand, (3.7) implies that

$$
\left\|P\left(\left(a_{k+\tau}\right)_{k, \tau}\right)\right\|_{\infty}^{\rho} \leq(B+4)\left\|\left(a_{k+\tau}\right)_{k, \tau}\right\|,
$$


hence $P: c_{0}(\mathbb{Z}+\widetilde{D}) \rightarrow C_{0}^{\rho}(\mathbb{R})$ is well defined, clearly linear and continuous.

Observe that clearly

$$
\left\{\varphi_{k+\tau}, k \in \mathbb{Z}, \tau \in \widetilde{D}\right\} \subseteq P\left(c_{0}(\mathbb{Z}+\widetilde{D})\right) .
$$

If $f \in C_{0}^{\rho}(\mathbb{R})$, given $\varepsilon>0$, there exists $g \in C(\mathbb{R})$ with compact support such that $\|f-g\|_{\infty}^{\rho}<\frac{\varepsilon}{2}$. Let us assume that $\operatorname{supp}(g) \subseteq[-M, M]$, $M \in \mathbb{N}$ and consider $\varphi \in \operatorname{span}\left\{\varphi_{k+\tau}, k \in \mathbb{Z}, \tau \in \widetilde{D}\right\} \subseteq P\left(c_{0}(\mathbb{Z}+\widetilde{D})\right)$, $\operatorname{supp}(\varphi) \subseteq[-M-1, M+1]$, such that $\|g-\varphi\|_{\infty}<\frac{\varepsilon}{2 \max _{x \in[-M-1, M+1]} \rho(x)}$. Then $\|f-\varphi\|_{\infty}^{\rho}<\|f-g\|_{\infty}^{\rho}+\|g-\varphi\|_{\infty}^{\rho}<\frac{\varepsilon}{2}+\sup _{x \in[-M-1, M+1]}|g(x)-\varphi(x)| \rho(x)<\varepsilon$ and therefore $P$ has dense range.

Finally we observe that

$$
P \circ B_{w}\left(\left(a_{k+\tau}\right)_{k, \tau}\right)=\sum_{n=0}^{\infty} \frac{1}{2^{n}}\left(\sum_{k \in \mathbb{Z}, \tau \in D_{n}} \frac{a_{k+\tau+1}}{\rho(k+1)} \varphi_{k+\tau}\right) .
$$

On the other hand, for every $x \in \mathbb{R}$,

$$
\begin{aligned}
& T_{1} \circ P\left(\left(a_{k+\tau}\right)_{k, \tau}\right)(x)=\sum_{n=0}^{\infty} \frac{1}{2^{n}}\left(\sum_{k \in \mathbb{Z}, \tau \in D_{n}} \frac{a_{k+\tau}}{\rho(k)} \varphi_{k+\tau}(x+1)\right)= \\
& \sum_{n=0}^{\infty} \frac{1}{2^{n}}\left(\sum_{k \in \mathbb{Z}, \tau \in D_{n}} \frac{a_{k+\tau}}{\rho(k)} \varphi_{k+\tau-1}(x)\right)=\sum_{n=0}^{\infty} \frac{1}{2^{n}}\left(\sum_{h \in \mathbb{Z}, \tau \in D_{n}} \frac{a_{h+\tau+1}}{\rho(h+1)} \varphi_{h+\tau}(x)\right) .
\end{aligned}
$$

Then $P \circ B_{w}=T_{1} \circ P$.

Theorem 3.2. Let $\mathcal{T}$ be the translation semigroup on $C_{0}^{\rho}(\mathbb{R})$, where $\rho$ is an admissible weight function and $\sup _{k \in \mathbb{Z}} \rho(k+1) / \rho(k)<\infty$. Then $\mathcal{T}$ is frequently hypercyclic on $C_{0}^{\rho}(\mathbb{R})$ if and only if there exist a sequence $(M(p))_{p \in \mathbb{N}}$ of positive real numbers tending to $\infty$ and a sequence $\left(E_{p}\right)_{p \in \mathbb{N}}$ of subsets of $\mathbb{N}$ such that:

(a) for any $p \geq 1$, $\underline{\operatorname{dens}}\left(E_{p}\right)>0$,

(b) for any distinct $p, q \geq 1,\left(E_{p}+\llbracket-p, p \rrbracket\right) \cap\left(E_{q}+\llbracket-q, q \rrbracket\right)=\emptyset$,

(c) for any $p \geq 1, \lim _{n \rightarrow \infty, n \in E_{p}} \rho(n)=0$,

(d) for any $p, q \geq 1$ and any $n \in E_{p}$ and $m \in E_{q}$ with $m \neq n$,

$$
\rho(m-n) \leq \frac{1}{M(p) M(q)}
$$

Moreover, one can replace "there exist a sequence $(M(p))_{p \in \mathbb{N}}$ and a sequence $\left(E_{p}\right)_{p \in \mathbb{N}}$ " by "for any sequence $(M(p))_{p \in \mathbb{N}}$ there exists a sequence $\left(E_{p}\right)_{p \in \mathbb{N}}$ ". 
Proof. Since $\rho$ is an admissible weight function $\sup _{k \in \mathbb{Z}} \rho(k) / \rho(k+1)<\infty$, there exists $M>1$ such that for every $k \in \mathbb{Z}$,

$$
M^{-1} \leq \frac{\rho(k+1)}{\rho(k)} \leq M
$$

As a consequence we get for every $n \in \mathbb{N}$ we get, if $k \in \llbracket 0, p \rrbracket$,

$$
\rho(k+n)=\frac{\rho(k+n)}{\rho(k-1+n)} \ldots \frac{\rho(n+1)}{\rho(n)} \cdot \rho(n) \leq M^{k} \rho(n)
$$

and if $k \in \llbracket-p, 0 \rrbracket$,

$$
\rho(k+n)=\frac{\rho(k+n)}{\rho(k+1+n)} \cdots \frac{\rho(n-1)}{\rho(n)} \cdot \rho(n) \leq M^{|k|} \rho(n),
$$

thus for every $k \in \llbracket-p, p \rrbracket$,

$$
\rho(k+n) \leq M^{|k|} \rho(n) .
$$

On the other hand, by (1.1), there exist constants $0<A<B$ such that for every $x \in[k, k+1]$,

$$
A \rho(k) \leq \rho(x) \leq B \rho(k+1) \leq B M \rho(k) .
$$

If we now define $\tilde{\rho}(x)=\rho([x])$ for $x \in \mathbb{R}$, then there exist constants $M_{1}, M_{2}>0$ such that

$$
M_{1}\|f\|_{\infty}^{\tilde{\rho}} \leq\|f\|_{\infty}^{\rho} \leq M_{2}\|f\|_{\infty}^{\tilde{\rho}},
$$

so $\|\cdot\|_{\infty}^{\widetilde{\rho}}$ is an equivalent norm to $\|\cdot\|_{\infty}^{\rho}$. Therefore, without loss of generality we can assume in the following that $\rho(x)=\rho([x])$ for every $x \in \mathbb{R}$.

By the results in $[16,26]$, it is known that $\mathcal{T}$ is a frequently hypercyclic semigroup if and only if $T_{1}$ is a frequently hypercyclic operator. Since $T_{1}$ is quasiconjugate to the operator $B_{w}$ defined as in Lemma 3.1 and $B_{w}$ is quasiconjugate to $T_{1}$, by [24, Proposition 9.4] we find that $\mathcal{T}$ is frequently hypercyclic if and only if $B_{w}$ is frequently hypercyclic, hence, by Corollary 2.2, if and only if there exist a sequence $(M(p))_{p \in \mathbb{N}}$ of positive real numbers tending to $\infty$ and a sequence $\left(E_{p}\right)_{p \in \mathbb{N}}$ of subsets of $\mathbb{N}$ (or equivalently, for any $(M(p))_{p \in \mathbb{N}}$ there exists $\left.\left(E_{p}\right)_{p \in \mathbb{N}}\right)$ such that

(a1) for any $p \geq 1$, $\underline{\operatorname{dens}}\left(E_{p}\right)>0$,

(b1) for any distinct $p, q \geq 1,\left(E_{p}+W_{p}\right) \cap\left(E_{q}+W_{q}\right)=\emptyset$,

(c1) for every $p \geq 1$ and every $s \in W_{p}$,

$$
\lim _{n \rightarrow \infty, n \in E_{p}} w_{s} \ldots w_{s+n-1}=\lim _{n \rightarrow \infty, n \in E_{p}} \frac{\rho(s)}{\rho(s+n)}=\infty,
$$


(d1) for any $p, q \geq 1, n \in E_{p}$ and $m \in E_{q}$ with $m \neq n$, and $t \in W_{q}$,

$$
\frac{w_{m-n+t} \ldots w_{m+t-1}}{w_{t} \ldots w_{t+m-1}}=\frac{\rho(m-n+t)}{\rho(t)} \leq \frac{1}{M(p) M(q)}
$$

where $W_{p}=\llbracket-p, p \rrbracket+\widetilde{D}_{p}$.

Clearly (a1)-(d1) imply (a)-(d) simply by observing that $\llbracket-p, p \rrbracket \subseteq W_{p}$, and in particular $0 \in W_{p}$, for every $p \in \mathbb{N}$.

Conversely, assume that (a)-(d) hold. Passing to a subsequence of $\left(E_{p}\right)_{p \in \mathbb{N}}$ if necessary, we can choose $(M(p))_{p \in \mathbb{N}}$ such that

$$
\lim _{p \rightarrow \infty} \frac{M(p) \cdot \min \{\rho(k): k \in \llbracket-p, p \rrbracket\}}{M^{p}}=\infty .
$$

If

$$
\left(E_{p}+W_{p}\right) \cap\left(E_{q}+W_{q}\right) \neq \emptyset,
$$

then there exist $n \in E_{p}, s \in \llbracket-p, p \rrbracket, \sigma \in \widetilde{D}, m \in E_{q}, t \in \llbracket-q, q \rrbracket$ and $\tau \in \widetilde{D}$, such that

$$
n+s+\sigma=m+t+\tau
$$

taking integer parts yields $n+s=m+t$, hence $\left(E_{p}+\llbracket-p, p \rrbracket\right) \cap\left(E_{q}+\llbracket-q, q \rrbracket\right)$ $\neq \emptyset$. Thus $p=q$. So (b1) is satisfied.

By (3.9) and (c), we get $\lim _{n \rightarrow \infty, n \in E_{p}} \rho(n+k)=0$ for every $k \in \llbracket-p, p \rrbracket$, and (c1) follows by observing that for every $s \in W_{p}$ there exists $k \in \llbracket-p, p \rrbracket$ such that $\rho(n+s)=\rho(n+k)$.

Let $p, q \geq 1$ and $n \in E_{p}, m \in E_{q}, m \neq n$. By (3.9) and (d), for every $k \in \llbracket-q, q \rrbracket$,

$$
\begin{aligned}
\frac{\rho(m-n+k)}{\rho(k)} & \leq \frac{\rho(m-n)}{\rho(k)} M^{|k|} \leq \frac{M^{q}}{\min \{\rho(k): k \in \llbracket-q, q \rrbracket\} M(p) M(q)} \\
& \leq \frac{1}{K(p) K(q)}
\end{aligned}
$$

where

$$
K(q)=\min \left\{\frac{M(q) \cdot \min \{\rho(k): k \in \llbracket-q, q \rrbracket\}}{M^{q}}, M(q)\right\} .
$$

We get (d1) by observing that for every $t \in W_{q}$ there exists $k \in \llbracket-q, q \rrbracket$ such that

$$
\frac{\rho(m-n+t)}{\rho(t)}=\frac{\rho(m-n+k)}{\rho(k)} .
$$

Remark 3.3. As an immediate consequence, if $\rho$ is an admissible weight function on $\mathbb{R}$ such that $\sup _{k \in \mathbb{Z}} \rho(k+1) / \rho(k)<\infty$ and we set $w_{k}=$ $\rho(k) / \rho(k+1)$ for $k \in \mathbb{Z}$, then, by the characterization given in $[12$, Theorem $9]$ and by Theorem $3.2, B_{w}$ is frequently hypercyclic on $c_{0}(\mathbb{Z})$ if and only if the translation semigroup is frequently hypercyclic on $C_{0}^{\rho}(\mathbb{R})$. 
Proposition 3.4. If the translation semigroup $\mathcal{T}$ is mixing (equivalently chaotic) on $C_{0}^{\rho}(\mathbb{R})$, then it is frequently hypercyclic.

Proof. As is proved in [13, 27], chaos and mixing are equivalent properties for the translation $C_{0}$-semigroup on $C_{0}^{\rho}(\mathbb{R})$, and this happens if and only if $\lim _{x \rightarrow \pm \infty} \rho(x)=0$.

As already observed, it is enough to prove that $T_{1}$ satisfies the Frequent Hypercyclicity Criterion for operators (see [14]). Let $X_{0}=\operatorname{span}\left\{\varphi_{k+\tau}: k \in\right.$ $\mathbb{Z}, \tau \in \widetilde{D}\}$, where $\varphi_{k+\tau}$ is defined in (3.1). Every continuous function on $\mathbb{R}$ with dense support can be approximated in the uniform norm with elements of $X_{0}$, and therefore $X_{0}$ is dense in $C_{0}^{\rho}(\mathbb{R})$. Moreover, if we define $S: X_{0} \rightarrow X_{0}$ by $S f(x)=f(x-1)$, it is clear that $T_{1} S f=f$.

Let us prove that $\sum_{n=1}^{\infty} T_{1}^{n} f$ and $\sum_{n=1}^{\infty} S^{n} f$ are unconditionally convergent for all $f \in X_{0}$. It is enough to consider $f=\varphi_{k+\tau} \in X_{0}$; then $\operatorname{supp}(f) \subseteq[a, b]$ with $b-a \leq 2$, and for every $n \in \mathbb{N}$,

$$
\operatorname{supp}\left(T_{1}^{n}(f)\right) \subseteq[a-n, b-n]
$$

thus for every $n, m \in \mathbb{N}$, if $\operatorname{supp}\left(T_{1}^{n} f\right) \cap \operatorname{supp}\left(T_{1}^{m} f\right) \neq \emptyset$, then it is immediate that $|n-m| \leq|b-a| \leq 2$, hence either $m=n$, or $m=n \pm 1$, or $m=n \pm 2$. This implies that if $J \subseteq \mathbb{N}$ is finite, then

$$
\left\|\sum_{n \in J} T_{1}^{n} f\right\|_{\infty}^{\rho} \leq 4 \sup _{n \in J}\left\|T_{1}^{n} f\right\|_{\infty}^{\rho} .
$$

Let $\varepsilon>0$ and let $M>0$ be such that $\rho(x)<\varepsilon$ for every $|x|>M$. For every finite set $F \subseteq \mathbb{N} \cap] M+b, \infty[$ and every $x \in[a, b]$ and $n \in F$, we have $|x-n|=n-x>M$, so

$$
\left\|\sum_{n \in F} T_{1}^{n} f\right\|_{\infty}^{\rho} \leq 4 \sup _{x \in \mathbb{R}, n \in F}|f(x+n) \rho(x)|=2 \sup _{x \in[a, b], n \in F}|f(x) \rho(x-n)| \leq 2 \varepsilon .
$$

The argument for $\sum_{n=1}^{\infty} S^{n} f$ is similar.

Remark 3.5. The converse of the previous proposition does not hold. Indeed, let $\left(w_{k}\right)_{k \in \mathbb{Z}}$ be one of the sequences of weights constructed in [12] such that $B_{w}$ is frequently hypercyclic on $c_{0}(\mathbb{Z})$ and $w_{1} \cdots w_{k}=1$ for infinitely many $k$. Define $\rho(k)=\left(w_{1} \cdots w_{k}\right)^{-1}$ if $k \geq 1, \rho(k)=w_{k} w_{k+1} \cdots w_{0}$ if $k \leq 0$, and $\rho(x)=\rho([x])$ for any $x \in \mathbb{R}$. Then $\sup _{k \in \mathbb{Z}} \rho(k+1) / \rho(k)<\infty$, due to the fact that $1 / 2 \leq w_{k} \leq 2$ for all $k \in \mathbb{Z}$, as shown in [12]. By Remark 3.3 the translation semigroup is frequently hypercyclic on $C_{0}^{\rho}(\mathbb{R})$, while clearly it is not mixing, since $\rho(k)=1$ for infinitely many $k$. 
Finally, set $J=\mathbb{Z}_{+}+\widetilde{D}$ and consider the partition $J=\bigcup_{n \geq 0} J_{n}$ where $J_{0}=\{0\}$ and

$$
J_{n}=\left\{h+D_{n-h}: h=0,1, \ldots, n\right\} \quad \text { for } n \geq 1 .
$$

Define an order on $J$ assuming that the elements $J_{k}$ are earlier than those in $J_{n}$ if $0 \leq k<n$, and within each $J_{n}$ we keep the usual order.

The system $\left(\psi_{i}\right)_{i \in J}$, where $\psi_{0}(x):=\max \{0,1-x\}$ and $\psi_{k+\tau}=\varphi_{k+\tau}$ if $\tau \neq 0$ or $k \neq 0$, is a Schauder basis on $C_{0}([0, \infty[)$.

Reasoning analogously to the case of translation semigroups in $C_{0}^{\rho}(\mathbb{R})$, we also get the following characterization of frequently hypercyclic translation semigroups on $C_{0}^{\rho}([0, \infty[)$ :

Theorem 3.6. Let $\mathcal{T}$ be the translation semigroup on $C_{0}^{\rho}([0, \infty[)$, where $\rho$ is an admissible weight function and $\sup _{k \in \mathbb{N}} \rho(k+1) / \rho(k)<\infty$. Then $\mathcal{T}$ is frequently hypercyclic on $C_{0}^{\rho}([0, \infty[)$ if and only if there exist a sequence $(M(p))_{p \in \mathbb{N}}$ of positive real numbers tending to $\infty$ and a sequence $\left(E_{p}\right)_{p \in \mathbb{N}}$ of subsets of $\mathbb{N}$ such that:

(a) for any $p \geq 1$, $\underline{\operatorname{dens}}\left(E_{p}\right)>0$,

(b) for any distinct $p, q \geq 1,\left(E_{p}+\llbracket 0, p \rrbracket\right) \cap\left(E_{q}+\llbracket 0, q \rrbracket\right)=\emptyset$,

(c) for every $p \geq 1, \lim _{n \rightarrow \infty, n \in E_{p}} \rho(n)=0$,

(d) for any $p, q \geq 1$ and any $n \in E_{p}$ and $m \in E_{q}$ with $m>n$,

$$
\rho(m-n) \leq \frac{1}{M(p) M(q)},
$$

Moreover, one can replace "there exist a sequence $(M(p))_{p \in \mathbb{N}}$ and a sequence $\left(E_{p}\right)_{p \in \mathbb{N}}$ " by "for any sequence $(M(p))_{p \in \mathbb{N}}$ there exists a sequence $\left(E_{p}\right)_{p \in \mathbb{N}}$ ".

The final part of the paper will be devoted to characterizing frequently hypercyclic semigroups on $L_{p}^{\rho}(\mathbb{R})$. Also in this case we will first establish a relation between the discrete and the continuous cases. We recall that the relation between the discrete and the continuous cases for Devaney chaos was studied in [8] and for distributional chaos in [7]. The following lemma follows immediately from the conjugacy of the backward shift $B$ on $\ell_{p}^{v}=\left\{\left(x_{k}\right)_{k \in \mathbb{Z}}\right.$ : $\left.\sum_{k \in \mathbb{Z}}\left|x_{k}\right|^{p} v_{k}<\infty\right\}$ and the weighted backward shift $B_{w}$ on $\ell_{p}$ where $w_{k}=$ $\left(v_{k} / v_{k+1}\right)^{1 / p}$ for $k \in \mathbb{Z}$, and from the characterization of frequently hypercyclic weighted backward shifts on $\ell_{p}$ proved in [12, Theorem 3].

Lemma 3.7. Let $v=\left(v_{k}\right)_{k \in \mathbb{Z}}$ be a sequence of strictly positive weights such that $\left(v_{k} / v_{k+1}\right)_{k}$ is bounded. Then the backward shift operator $B$ is frequently hypercyclic on $\ell_{p}^{v}$ if and only if $\sum_{k \in \mathbb{Z}} v_{k}<\infty$. 
Theorem 3.8. Let $\rho$ be an admissible weight function on $\mathbb{R}$. If the translation semigroup $\mathcal{T}$ is frequently hypercyclic on $L_{p}^{\rho}(\mathbb{R})$, then the backward shift operator $B$ is frequently hypercyclic on $\ell_{p}^{v}$, where $v_{k}=\rho(k)$ for all $k \in \mathbb{Z}$.

Proof. Since $\rho$ is an admissible weight function, by (1.1) there exist $A, B \geq 0$ such that $A \rho(k) \leq \rho(t) \leq B \rho(k+1)$ for all $t \in[k, k+1]$. If $\left(T_{t}\right)_{t \geq 0}$ is frequently hypercyclic, then $T_{1}$ is frequently hypercyclic [16]. Hence there exists $f \in L_{p}^{\rho}(\mathbb{R})$ such that for all $g \in L_{p}^{\rho}(\mathbb{R})$ and for all $\varepsilon>0$,

$$
\underline{\operatorname{dens}}\left(\left\{n \in \mathbb{N}:\left\|T_{1}^{n} f-g\right\|<\varepsilon\right\}\right)>0 .
$$

Since $f \in L_{p}^{\rho}(\mathbb{R})$ we have $|f| \rho^{1 / p} \in L_{p}([k, k+1]) \subseteq L_{1}([k, k+1])$ for every $k \in \mathbb{Z}$. As $\rho$ is strictly positive and locally bounded below by (1.1), we infer that $f \in L_{1}([k, k+1])$ for all $k \in \mathbb{Z}$. Therefore we can define $x_{k}=\int_{k}^{k+1} f(t) d t$ for all $k \in \mathbb{Z}$. We have

$$
\begin{aligned}
\sum_{k \in \mathbb{Z}}\left|x_{k}\right|^{p} \rho(k) & =\sum_{k \in \mathbb{Z}}\left|\int_{k}^{k+1} f(t) d t\right|^{p} \rho(k) \leq \sum_{k \in \mathbb{Z}} \int_{k}^{k+1}|f(t)|^{p} \rho(k) d t \\
& \leq \frac{1}{A} \sum_{k \in \mathbb{Z}} \int_{k}^{k+1}|f(t)|^{p} \rho(t) d t=\frac{1}{A}\|f\|_{p}^{p}<\infty .
\end{aligned}
$$

So $x=\left(x_{k}\right)_{k \in \mathbb{Z}} \in \ell_{v}^{p}$ with $v_{k}=\rho(k)$.

Let

$$
y=\left(0, \ldots, y_{-N}, \ldots, y_{0}, \ldots, y_{M}, 0, \ldots, 0\right)
$$

and let $\varepsilon>0$. Set $g=\sum_{k=-N}^{M} y_{k} \chi_{[k, k+1]} \in L_{p}^{\rho}(\mathbb{R})$. We will show that

$$
\left\{n \in \mathbb{N}:\left\|T_{1}^{n} f-g\right\|<A^{1 / p} \varepsilon\right\} \subseteq\left\{n \in \mathbb{N}:\left\|B^{n} x-y\right\|<\varepsilon\right\},
$$

and therefore

$$
\underline{\operatorname{dens}}\left(\left\{n \in \mathbb{N}:\left\|B^{n} x-y\right\|<\varepsilon\right\}\right)>0
$$

because $f$ is a frequently hypercyclic vector. We have

$$
\begin{aligned}
\left\|B^{n} x-y\right\|^{p} & =\sum_{k \in \mathbb{Z}}\left|x_{n+k}-y_{k}\right|^{p} \rho(k) \\
& \leq \frac{1}{A} \sum_{k \in \mathbb{Z}} \int_{k}^{k+1}|f(t+n)-g(t)|^{p} \rho(t) d t \leq \varepsilon^{p} .
\end{aligned}
$$

By the density of finite sequences in $\ell_{p}^{v}$ we conclude that $B$ is frequently hypercyclic.

Finally, we are able to characterize frequently hypercyclic translation semigroups on $L_{p}^{\rho}(\mathbb{R})$. 
Theorem 3.9. Let $\rho$ be an admissible weight function on $\mathbb{R}$. The following assertions are equivalent:

(1) The translation semigroup $\mathcal{T}$ is frequently hypercyclic on $L_{p}^{\rho}(\mathbb{R})$.

(2) $\sum_{k \in \mathbb{Z}} \rho(k)<\infty$.

(3) $\int_{-\infty}^{\infty} \rho(t) d t<\infty$.

(4) $\mathcal{T}$ is chaotic on $L_{p}^{\rho}(\mathbb{R})$.

(5) $\mathcal{T}$ satisfies the Frequent Hypercyclicity Criterion.

Proof. Observe that $(\rho(k) / \rho(k+1))_{k \in \mathbb{Z}}$ is bounded by the admissibility of $\rho$. By Theorem 3.8 and Lemma 3.7, we have $(1) \Rightarrow(2)$. The equivalence of $(2)$ and (3) follows from the properties of $\rho$, by comparing integrals and series. The equivalence of (4) and (5) can be proved with the same argument as in [26, Proposition 3.3]. (5) $\Rightarrow(1)$ is proved in [26, Theorem 2.2], while $(3) \Rightarrow(5)$ can be proved as in [26, Proposition 3.4].

With minor changes we also get a characterization of frequently hypercyclic translation semigroups on $L_{p}^{\rho}([0, \infty[)$ :

Theorem 3.10. Let $\rho$ be an admissible weight function on $[0, \infty[$. The following assertions are equivalent:

(1) The translation semigroup $\mathcal{T}$ is frequently hypercyclic on $L_{p}^{\rho}([0, \infty[)$.

(2) $\sum_{k \in \mathbb{N}} \rho(k)<\infty$.

(3) $\int_{0}^{\infty} \rho(t) d t<\infty$.

(4) $\mathcal{T}$ is chaotic on $L_{p}^{\rho}([0, \infty[)$.

(5) $\mathcal{T}$ satisfies the Frequent Hypercyclicity Criterion.

Acknowledgements. The authors thank the anonymous referee for the critical reading of the manuscript and for his constructive comments.

This work is supported by MEC and FEDERER, proyect MTM 201347093-P. The second author also acknowledges the support of a grant by the FPU Program of Ministerio de Educación and a travel grant from the Foundation Ferran Sunyer i Balaguer, and she thanks the Dipartimento di Matematica e Fisica "E. De Giorgi" (Lecce, Italy) for the hospitality during her stay. 


\section{References}

[1] A. A. Albanese, X. Barrachina, E. M. Mangino, and A. Peris, Distributional chaos for strongly continuous semigroups of operators, Comm. Pure Appl. Anal. 12 (2013), 2069-2082.

[2] J. Aroza, T. Kalmes, and E. M. Mangino, Chaotic $C_{0}$-semigroups induced by semiflows in Lebesgue and Sobolev spaces, J. Math. Anal. Appl. 412 (2014), 77-98.

[3] C. Badea and S. Grivaux, Unimodular eigenvalues uniformly distributed sequences and linear dynamics, Adv. Math. 211 (2007), 766-793.

[4] J. Banasiak, M. Lachowicz, and M. Moszyński, Semigroups for generalized birth-and-death equations in $l^{p}$ spaces, Semigroup Forum 73 (2006), 175-193.

[5] J. Banasiak, M. Lachowicz, and M. Moszyński, Chaotic behaviour of semigroups related to the process of gene amplification-deamplification with cell proliferation, Math. Biosci. 206 (2007), 200-215.

[6] J. Banasiak and M. Moszyński, Dynamics of birth-and-death processes with proliferation-stability and chaos, Discrete Contin. Dynam. Systems 29 (2011), 67-79.

[7] X. Barrachina and A. Peris, Distributionally chaotic translation semigroups, J. Difference Equ. Appl. 18 (2012), 751-761.

[8] F. Bayart and T. Bermúdez, Semigroups of chaotic operators, Bull. London Math. Soc. 41 (2009), 823-830.

[9] F. Bayart and S. Grivaux, Frequently hypercyclic operators, Trans. Amer. Math. Soc. 358 (2006), 5083-5117.

[10] F. Bayart and S. Grivaux, Invariant Gaussian measures for operators on Banach spaces and linear dynamics, Proc. London Math. Soc. (3) 94 (2007), 181-210.

[11] F. Bayart and É. Matheron, Dynamics of Linear Operators, Cambridge Univ. Press, Cambridge, 2009.

[12] F. Bayart and I. Z. Ruzsa, Difference sets and frequently hypercyclic weighted shifts, Ergodic Theory Dynam. Systems 35 (2015), 691-709. 
[13] T. Bermúdez, A. Bonilla, J. A. Conejero, and A. Peris, Hypercyclic, topologically mixing and chaotic semigroups on Banach spaces, Studia Math. 170 (2005), 57-75.

[14] A. Bonilla and K.-G. Grosse-Erdmann, Frequently hypercyclic operators and vectors, Ergodic Theory Dynam. Systems 27 (2007), 383-404; Erratum: ibid. 29 (2009), 1993-1994.

[15] J. A. Conejero and E. M. Mangino, Hypercyclic semigroups generated by Ornstein-Uhlenbeck operators, Mediterr. J. Math. 7 (2010), 101-109.

[16] J. A. Conejero, V. Müller, and A. Peris, Hypercyclic behaviour of operators in a hypercyclic $C_{0}$-semigroup, J. Funct. Anal. 244 (2007), 342-348.

[17] J. A. Conejero and A. Peris, Hypercyclic translation $C_{0}$-semigroups on complex sectors, Discrete Contin. Dynam. Systems 25 (2009), 1195-1208.

[18] M. De la Rosa, L. Frerick, S. Grivaux, and A. Peris, Frequent hypercyclicity, chaos, and unconditional Schauder decompositions, Israel J. Math. 190 (2012), 389-399.

[19] R. deLaubenfels and H. Emamirad, Chaos for functions of discrete and continuous weighted shift operators, Ergodic Theory Dynam. Systems 21 (2001), 1411-1427.

[20] W. Desch, W. Schappacher, and G. F. Webb, Hypercyclic and chaotic semigroups of linear operators, Ergodic Theory Dynam. Systems 17 (1997), 793-819.

[21] H. Emamirad, G. Ruiz Goldstein and J. A. Goldstein, Chaotic solution for the Black-Scholes equation, Proc. Amer. Math. Soc. 140 (2012), 2043-2052.

[22] S. Grivaux, A probabilistic version of the frequent hypercyclicity criterion, Studia Math. 176 (2006), 279-290.

[23] K.-G. Grosse-Erdmann, Hypercyclic and chaotic weighted shifts, Studia Math. 139 (2000), 47-68.

[24] K.-G. Grosse-Erdmann and A. Peris Manguillot, Linear Chaos, Universitext, Springer London, London, 2011. 
[25] T. Kalmes, Hypercyclic, mixing, and chaotic $C_{0}$-semigroups induced by semiflows, Ergodic Theory Dynam. Systems 27 (2007), 1599-1631.

[26] E. M. Mangino and A. Peris, Frequently hypercyclic semigroups, Studia Math. 202 (2011), 227-242.

[27] M. Matsui, M. Yamada, and F. Takeo, Supercyclic and chaotic translation semigroups, Proc. Amer. Math. Soc. 131 (2003), 3535-3546; Erratum: ibid. 132 (2004), 3751-3752.

[28] M. Murillo Arcila and A. Peris, Strong mixing measures for $C_{0^{-}}$ semigroups, Rev. R. Acad. Cienc. Exactas Fís. Nat. Ser. A Mat. 109 (2015), 101-116.

[29] Z. Semadeni, Schauder Bases in Banach Spaces of Continuous Functions, Lecture Notes in Math. 918, Springer, Berlin, 1982. 